\title{
Minocycline for Schizophrenia: A critical review
}

\author{
Tsuyoshi Miyaoka \\ Department of Psychiatry, Shimane University School of Medicine, Izumo, Japan \\ Email: miyanyan@med.shimane-u.ac.jp
}

Received 9 September 2012; revised 9 October 2012; accepted 18 October 2012

\begin{abstract}
Minocycline, an antibiotic of the tetracycline family, has been shown to display neurorestoractive or neuroprotective properties in various models of neurodegenerative diseases. In particular, it has been shown to delay motor alterations, inflammation and apoptosis in models of Huntington's disease, amyotrophic lateral sclerosis and Parkinson's disease. Despite controversies about its efficacy, the relative safety and tolerability of minocycline have led to the launching of various clinical trials. Previously, we reported the antipsychotic effects of minocycline in patients with schizophrenia. In a pilot investigation, we administered minocycline as an open-label adjunct to antipsychotic medication to patients with schizophrenia. The results of this trial suggested that minocycline might be a safe and effective adjunct to antipsychotic medications, and that augmentation with minocycline may prove to be a viable strategy for "boosting" antipsychotic efficacy and for treating schizophrenia. Recently, in randomized double-blind placebo-controlled clinical trials, the addition of minocycline to treatment as usual early in the course of schizophrenia predominantly improves negative symptoms. The present review summarizes the available data supporting the clinical testing of minocycline for patients with schizophrenia. In addition, we extend our discussion to the potential applications of minocycline for combining this treatment with cellular and molecular therapy.
\end{abstract}

Keywords: Minocycline; Schizophrenia; Apoptopsis; Inflammation

\section{INTRODUCTION}

There is a dramatic need of neuroprotective treatments for neurodegenerative disorders. This is crucial for lethal disorders such as amyotrophic lateral sclerosis (ALS) and Huntington's disease (HD) for which no pharmacological or other efficient treatment is available yet. Recent experimental evidence from in vitro and in vivo models of degeneration points out that minocycline could have beneficial properties independent of their initial antibactericid effects [1]. This is of high interest because these molecules have been clinically well known and have a long history of safe use.

Schizophrenia is a complex disorder characterized by profound disturbances of perception, cognition, emotion and social function. It affects approximately $0.5 \%-1.0 \%$ of the general population. The treatment of patients suffering from schizophrenia who experience minimal or no response to adequate doses of antipsychotics represents an enomous challenge to clinicians. These antipsychotic treatmen-resistant patients constitute up to $25 \%$ of all patients suffering from schizophrenia [2]. In such situations clinicians embark on therapeutic trials of alterative strategies that usually involve the addition of other agents to the standard antipsychotic treatment. We reported the antipsychotic effects of minocycline in patients with schizophrenia [3]. In pilot investigation, we administered minocycline as an open-label adjunct to antipsychotic medication to patients with schizophrenia [4]. Recently, in randomized double-blind placebo-controlled clinical trials, the addition of minocycline to treatment as usual early in the course of schizophrenia predominantly improves negative symptoms [4-6].

Based on recent experimental and clinical data, herein we review the preclinical and clinical potential of minocycline in schizophrenia.

\section{MINOCYCLINE: CLINICAL PHARMACOLOGY}

Minocycline is a broad-spectrum, second-generation semisynthetic antibiotic belonging to the tetracycline family, approved by the FDA and indicated (100 - 200 $\mathrm{mg}$ /day) for acne vulgaris, some sexually transmitted diseases and rheumatoid arthritis [7]. In rodents, minocycline readily crosses the blood-brain barrier with a rate of at least fivefold higher than doxycycline, another compound of the same family [8].

In humans, long-term treatment with minocycline up to $200 \mathrm{mg}$ /day is generally safe and well tolerated as demonstrated by tolerability tests and clinical trials in rheumatoid arthritis, acne vulgaris and HD [9]. In some rare cases, the treatment can cause reversible side effects 
such as liver toxicity, serum sickness, and systemic lupus erythematosus [10]. Given that chronic treatment increases the risk for adverse effects, liver and renal functions, as well as immunologic status, should be assessed before starting treatment and monitored regularly during the administration [11].

\section{MECHANISMS UNDERLYING MINOCYCLINE-INDUCED NEUROPROTECTION}

Almost all the beneficial effects of minocycline are related to an inhibitory activity on inflammation and/or apoptotic cell death, both phenomena being intimately related to neural degeneration. Several studies clearly indicate that minocycline is able to inhibit inflammation and more particularly microgliosis. Morphologically, minocycline reduce the proliferation/activation of resting microglial cells [12].

\subsection{Inhibition of Microglial Activation}

Evidence that minocycline prevents microglial activation was first provided by Yrjanheikki and colleagues [13] in model of forebrain ischemia in gerbils. Decreased microglial activation by minocycline has since been reported in models of neurodegenerative disorders [14]. The expression of several molecules associated with microglial activation, including caspase 1 (interleukin-1bconverting enzyme) and inducible nitric oxide synthase, reduced expression after treatment with minocycline [15]. Nevertheless, these reports do not discriminate between a direct effect of minocycline in the inhibition of microglial activation or an indirect effect through reduced neurodegeneration and, consequently, less microglial activation.

Whether minocycline has a direct or an indirect effect on microglial activation was investigated in tissue culture. Minocycline directly inhibited the proliferation and the activation state of cultured microglia [16]. Microglial activation in tissue culture contributed to glutamate excitotoxicity, and microglial activation was reduced by minocycline with associated alleviation of excitotoxicity [17].

That minocycline inhibits microglial activation has been exploited in the area of cell transplants and neurogenesis. A myeline mutation in the spinal cords of Long Evans Shaker rats produces myeline defects that are associated with progressive microglial activation. In this model, the transplantation of oligodendroglial progenitor cells during peak microglial activation did not lead to myelination because the grafted cells died promptly after transplantation. However, pretreatment of these animals with minocycline reduced microglial activation and resulted in cell survial and myelin formation by the implants [18]. Furthermore, hippocampal neurogenesis, a process that continutes in the adult brain, was impaired by microglial activation caused by lipopolysaccharide infusion into the brain, and this difient neurogenesis was restored by the systemic administration of minocycline in association with reduced microglial activation [19].

\subsection{Attenuation of Apoptosis}

Apoptosis and the release of apoptosis-inducing drug is a common mechanism of neurodegeneration. Minocycline reduces apoptosis of neurons and oligodendrocytes in various neural insults [20] and alleviates necroric cell death [21]. There is increasing evidence to suggest that the antiapoptotic effect is achieved through several mechanisms at the level of the mitochondrion. Minocycline stabilities mitochondria membranes and inhibits the mitochondrial permeability transition-mediate release of ctochrome c into the cytosol [22], which is a potent stimulus for the activation of caspase 9 and 3 and the induction of apoptosis. The stabilization of mitochondrial membranes also reduces the release into the cytoplasm of other factors that trigger both caspase-dependent and caspase-independent apoptoic pathways, including apoptosis-including factor and Smac/Diablo [23]. In cell culture, minocycline upregulates the anti-apoptoic factor Bcl-2, which then accumulates in mitochondria to antagonise the pro-apoptotic Bcl-2 family members Bax, Bak, and Bid [24]. Indeed, the downregulation of Bcl-2 by antisense oligonucleotides prevents the protective capacity of minocycline in vitro. Finally, in a cell model of Huntington's disease, minocycline inhibitede the generation of the proapoptoic protein, t-Bid [25].

\subsection{Supression of Free-Radical Production}

The generation of free radicals, leading to lipid and protein peroxidation and damage to membranes is also a common mechanism across a spectrum of disease. Minocycline depresses the release of oxygen radicals from various cell types, including leucocytes. As mentioned above, the production of nitric oxide is decreased by minocycline through an effect on nitric oxide synthase [26].

\subsection{Ihibition of MMPs}

Another mechanism for minocycline action that may account for its effect on various neurological disease is its inhibition of MMPs. Various MMPs are upregulate in neurological disorders. In which they can contribute to demyelination, neurotoxicity, and neuroinflammation [27]. Thus, it is relevant that minocycline is a direct inhibitor of MMP enzymatic activity [28] and can also reduce the production of MMPs by leucocytes [29]. An effect on MMPs can also affect the transmigration of leucocytes into the CNS [30], thereby reducing neurounflammation further. Various inflammmatorycell subsets 
can disrupt CNS functions and produce toxic effects when present in the CNS in large numbers.

\subsection{Changes in Leucocyte Function}

Besides inhibiting MMPs, leukocyte migration, and neuroinflammation in the the nervous system, minocycline also has a direct effect on the activity of leucocytes. Kloppenburg and colleagues [31] noted that minocycline inhibited T-cell proliferation and reduced their oroduction of inflammatory cytokines. By contrast, the addition of minocyline to activated monocytes led to a dose-dependent increase in the production of tumor necrosis factor $\alpha$ and interleukin 6 [32].

\subsection{Other Mechanisms}

Other mechanisms may also contribute to the activity of minocycline. The drug is a $\mathrm{Ca}^{2+}$ cheltor and may sequester excess $\mathrm{Ca}^{2+}$ release after injury. The dcreasing of $\mathrm{Ca}^{2+}$ concentrations may prevent activation of calpains and preserve axonal integrity [15]. Firthermore, minocycline can inhibit the activation of p38 mitogen-activated protein (MAP) kinase and thus influence multiple processes that would otherwise reduce cell integrity. The antibiotic activity of minocycline is unlikely to accont fot its effectiveness in neurological diseases, since infection is not a component of these animal models, and because minocycline derivatives without antimicrobial action can also affect some of the above processes [17].

In summary, among several activites, minocycline impairs microglial activation, neuroinflammation, and apotosis, which are all common to many neurological diseases and schizophrenia [33,34]. The early appearance of these mediators of injury may help account for the apparent transient need for giving minocycline in the first few days after damage, such as in spinal-cord injury. Although many of the benefits of minocycline are likely to be derived from its action in the CNS, its effects on T cells and other leucocyte subsets could also happenin the periphery.

\section{MINOCYCLINE IN EXPERIMENTAL MODELS OF SCHIZOPHRENIA}

Levkovitz et al. indicated the effects of minocycline in an animal model of schizophrenia involving, the noncompetitive N-methyl-D-aspartate receptor antagonist (dizocilpine maleate; MK801) [35]. The effects of minocycline were compared to those of haloperidol, a dopamine antagonist used for the treatment of schizophrenia. The study protocol involved daily intraperitoneal injections of minocycline for three consecutive days. On the fourth day, the rats were injected with MK801 and assessed for visual-spatial memory (Morris water maze) and sensorimotor gating (acoustic startle response (ASR), and the PPI of the ASR). MK801 caused cognitive visuo-spatial memory deficits and changes in sensorimotor gating, similar to those evident in schizophrenia. Minocycline reversed these cognitive effects of MK801 and this effect was similar to that of haloperidol demonstrating protective properties against the cognitive effects of MK801.

Zhang et al. examined whether minocycline attenuates behavioral changes (e.g. acute hyperlocomotion and PPI deficits) in mice after the administration of MK-801 [36]. MK-801-induced hyperlocomotion was significantly attenuated by pretreatment with minocycline. Furthermore, PPI deficits after a single administration of MK801 were attenuated by pretreatment with minocycline $(10,20$, or $40 \mathrm{mg} / \mathrm{kg})$, in a dose-dependent manner. Moreover, in vivo microdialysis investigations in freemoving mice revealed that pretreatment with minocycline significantly attenuated the increase of extracellular dopamine (DA) levels in the frontal cortex and striatum after administration of MK-801, suggesting that the inhibition of MK-801-induced DA release by minocycline may, at least in part, be implicated in the mechanism of action of minocycline with respect to MK-801induced behavioral changes in mice. These finding led the authors to speculate that minocycline would be a potential therapeutic drug for schizophrenia.

Mizoguchi et al. reported the effect of minocycline on learning and memory in the novel object recognition test (NORT) and behavioral sensitization in mice that had been administered methamphetamine (METH) for 7 days [37]. When minocycline was administered intraperioneally once a day for seven consecutive days to mice that had previously been treated with METH for 7 days, minocycline ameliorated the METH-induced impairment of recognition memory in a dose-dependent manner, although the same treatment with minocycline had no effect on behavioral sensitization to METH. The administration of minocycline, together with METH, inhibited the development of METH-induced behavioral sensitization. The improvement in memory caused by minocycline was associated with an amelioration of the novelty-induced activation of extracellular signal-regulated kinase 1/2 in the prefrontal cortex of METH-treated mice. Since the dopaminergic overactivity engendered by METH has been associated with schizophrenic symptoms, the results of this study suggest that minocycline might be useful for the treatment of cognitive deficits in patients with METH induced psychosis or schizophrenia.

\section{POSSIBLE ANTIPSYCHOTIC EFFECTS OF MINOCYCLINE IN PATIENTS WITH SCHIZOPHRENIA: CASE REPORTS}

We reported two cases of acute schizophrenia that responded to minocycline [3]. In this report, minocycline 
was effective in treatment of acute schizophrenia with predominantly catatonic symptoms. Case 1: The patient was a 23-year-old male. He was diagnosed with catatonic schizophrenia. Haloperidol (4 - 20 mg/day) was started. One week later, his psychomotor excitement, auditory and catatonic stupor and persecutory delusions continued. Moreover his symptoms were complicated by severe pneumonia. A regimen of minocycline, $150 \mathrm{mg} /$ day was initiated, and haloperidol treatment was continued. Two weeks later, his psychiatric symptoms and pneumonia were recovered, and minocycline treatment was discontinued for a period of 1 week, which was followed by significant worsening of his psychiatric symptoms. Therefore, minocycline treatment was resumed and, within 3 days, a noticeable clinical improvement was observed. He was maintained on haloperidol and minocycline. 24 days later, the patient became practically symptom-free. His psychiatric symptoms were evaluated by the positive and negative symptom scale (PANSS) [38]. The clinical course is shown in Figure 1. Case 2: The patient was 61-year-old single male. At the age of 20, he experienced insomnia and auditory hallucinations and was diagnosed with schizophrenia. Since then he has had five psychiatric hospitalizations. In the last, he had been hospitalized for 4 years when he deteriorated and went into an autistic state. He was given $10 \mathrm{mg}$ of haloperidol and $2 \mathrm{mg}$ of risperidone per day for his psychosis. At the age of 60 , his mental state showed psychomotor excitement, catatonic stupor, and negativism. Moreover, a large decubitus had developed on his left hip. A regimen of minocycline, $150 \mathrm{mg}$ /day was initiated, and haloperidol and risperidone treatment were continued. Two weeks later, his decubitus was recovered, and minocycline treatment was discontinued for a period of 1 week, which was followed by significant worsening of his psychiatric symptoms. Therefore, minocycline treatment was resumed and, within 3 days, all noticeable clinical improvement was observed. He was maintained on haloperidol, risperidone, and a dose of $150 \mathrm{mg} /$ day of minocycline. 16 days later, the patient became practically symptom-free. He was maintained on minocycline plus haloperidol (3 mg/day) treatment, with no need for any additional drugs. His psychiatric symptoms were evaluated by the PANSS. The clinical course is shown in Figure 2. In these patient's, minocycline was effective in treatment of acute schizophrenia with predominantly by catatonic symptoms. At the remission of psychiatric symptoms, these patients did not suffer any infection for which minocycline indicated, and the use of minocycline is exclusive use for psychosis.

\section{CLINICAL TRIAL OF MINOCYCLINE IN PATIENTS WITH SCHIZOPHRENIA: OPEN-LABEL STUDY}

We examined the adjuvant therapeutic effect of minocycline added to stable regimens of antipsychotic medications in patients with schizophrenia [4]. Twenty-two patients with the diagnosis of schizophrenia (determined by the structural clinical interview of DSM-IV) [39] were recruited from outpatients and inpatients and entered into this study. The patients selected for study those not responding to their current medication or were treatment resistant. No patients had co-morbidity including alcohol and drug dependence, or depression. Psychiatric symptoms were rated by the PANSS.

After baseline assessment with the aforementioned instruments, minocycline treatment was initiated according
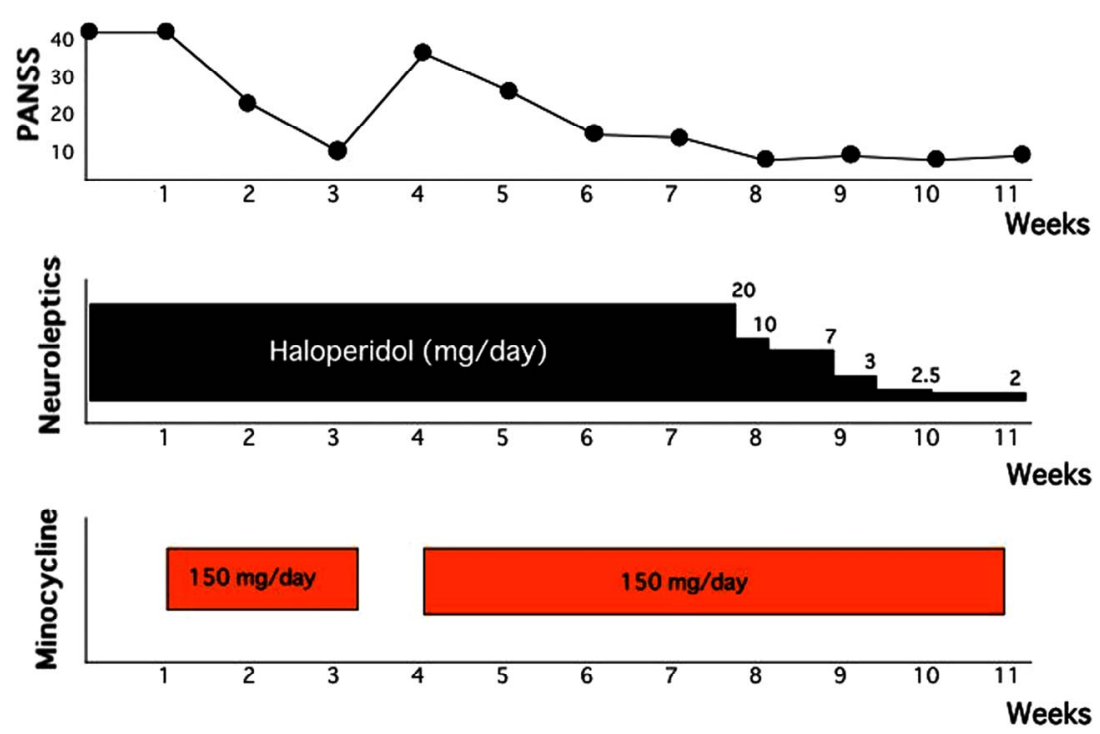

Figure 1. Clinical course of Case 1. 

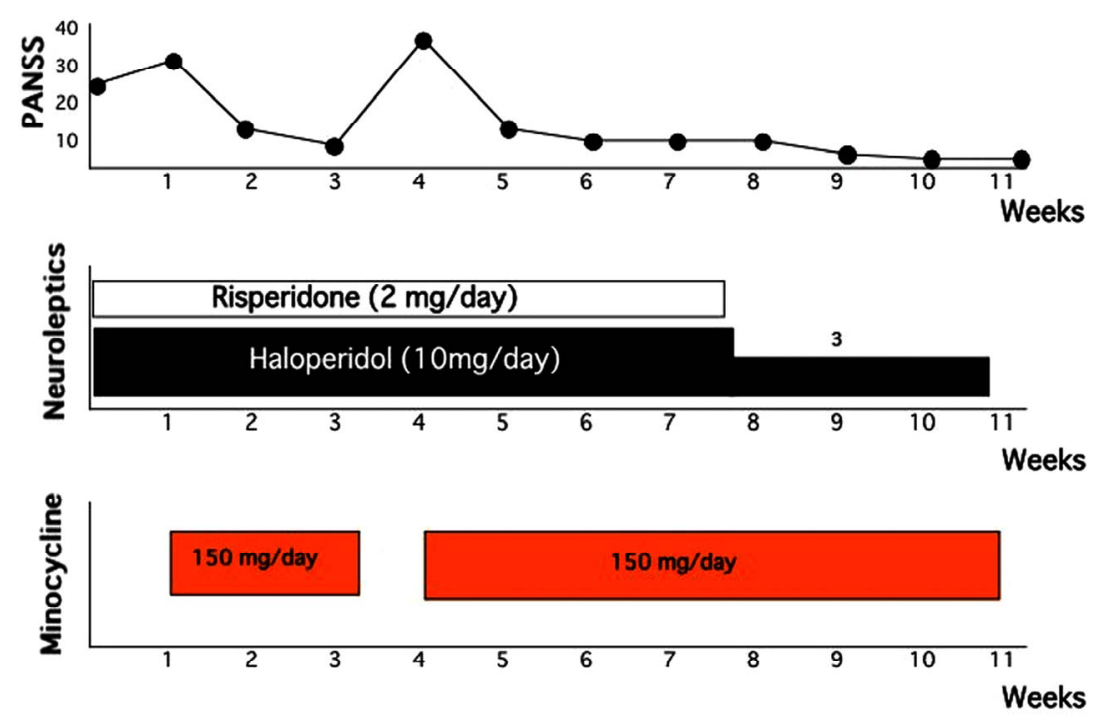

Figure 2. Clinical course of Case 2.

to the following titration schedule: $100 \mathrm{mg}$ orally twice daily for the first week, and $150 \mathrm{mg}$ orally three times daily from weeks 2 through 4 . All patients were taking atypical antipsychotic medications, including olanzapine, risperidone, quetiapine, and perospirone. The total duration of minocycline treatment was four weeks. The aforementioned clinical ratings were performed a total of three times: 1) at baseline before minocycline initiation, 2) at the end of open-label minocycline treatment, and 3) four weeks after minocycline treatment was discontinued. Patients were also assessed weekly during minocycline treatment to monitor compliance with medication and adverse events.

All patients tolerated the full dose of minocycline (150 mg three times daily) during the study. No adverse events were caused by minocycline treatment. The results of the outcome measure are presented in Figures 3-5). The PANSS positive symptoms subscale score was reduced by $40 \%$ at 4 weeks (mean score, $14.5 \pm 5.0$ ), and this reduction was maintained at the 4-week follow-up (mean score, $13.8 \pm 4.4$ ). The PANSS negative symptoms subscale score was reduced by $44 \%$ at 4 weeks (mean score, $14.4 \pm 5.3$ ), and this reduction was maintained at the 4-week follow-up (mean score, $14.1 \pm 4.9$ ). The PANSS general pychopathology subscale score was reduced by $52 \%$ at 4 weeks (mean score, $29.3 \pm 10.7$ ), and this reduction was maintained at the 4-week follow-up (mean score, $25.6 \pm 9.4$ ).

The present open-label study of adjunctive minocycline treatment of schizophrenia showed improvement in all PANSS subscales and presented preliminary evidence that minocycline may have a potential role in the treatment of schizophrenia. The small size and open nature of this study caution against any definite conclusions being drawn. However, the findings suggest that mino cycline
PANSS (positive)

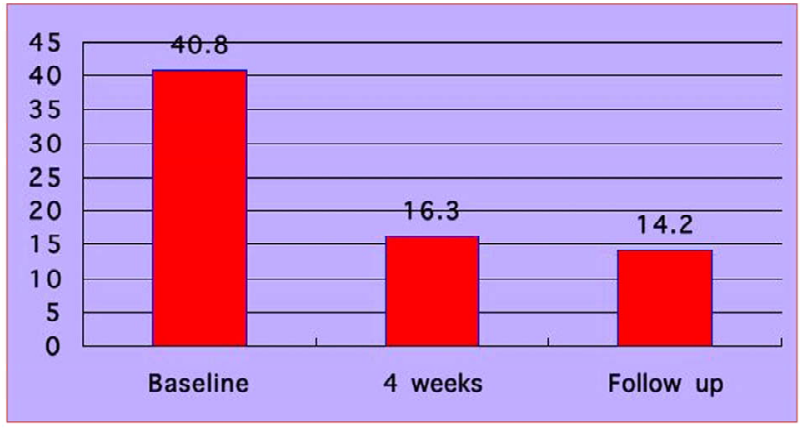

Miyaoka $\theta t$ al. Clin Neuropharmacol 2008

Figure 3. Patient data. PANSS (positive symptoms).

PANSS (negative)

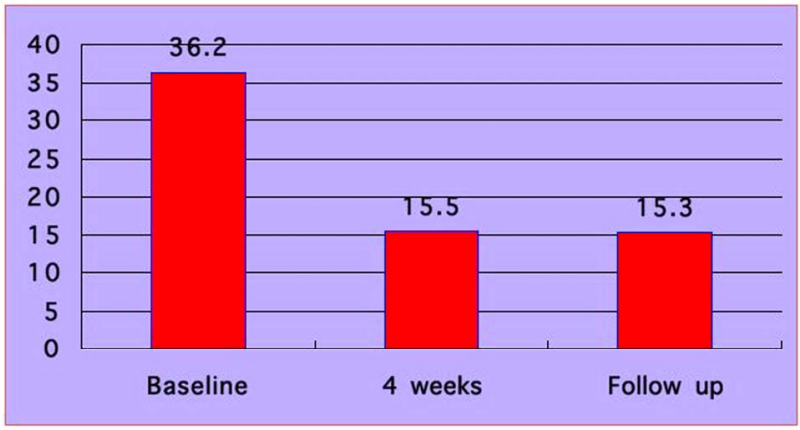

Miyaoka et al. Clin Neuropharmacol 2008

Figure 4. Patient data. PANSS (negative symptoms).

administration over 4 weeks in this subject group was feasible and safe, with no worsening of psychotic symptoms or neuropsychological function. The present data need to be confirmed in lager, randomized, and long term trials. 
PANSS (general)

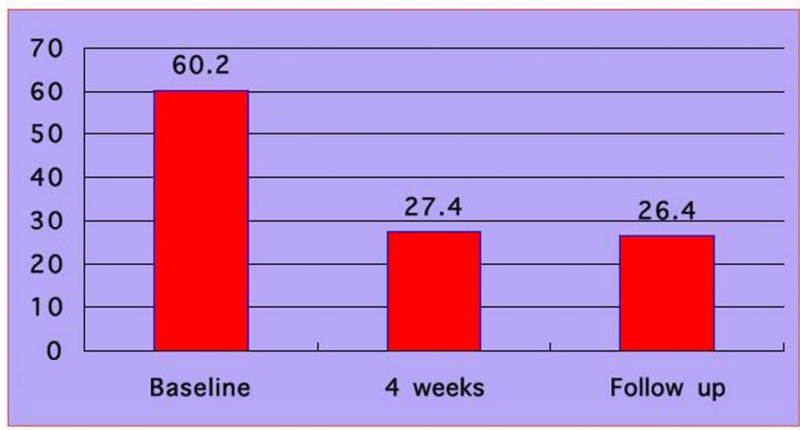

Miyaoka et al. Clin Neuropharmacol 2008

Figure 5. Patient Data. PANSS (general syndrome).

\section{CLINICAL TRIAL OF MINOCYCLINE IN PATIENTS WITH SCHIZOPHRENIA: DOUBLE-BLIND, RANDOMIZED STUDY}

Levkovitz et al. [5] reported, in longitudinal double-blind, randomized, placebo-controlled design, minocycline was well tolerated and it showed a beneficial effect on negative symptoms and general outcome, and a similar pattern was found for cognitive functioning, mainly in executive functions (working memory, cognitive shifting, and cognitive planning). They concluded minocycline treatment was associated with improvement in negative symptoms and executive functioning, both relative to frontal-lobe activity, and the findings support the beneficial effect of minocycline add-on therapy in early-phase schizophrenia.

Chaudhry et al. [6] investigated whether the addition of minocycline to treatment as usual (TAU) for 1 year in early psychosis would reduce negative symptoms compared with placebo. They concluded that the addition of minocycline to TAU early in the course of schizophrenia predominantly improves negative symptoms, and whether this is mediated by neuroprotective, anti-inflammatory or other actions is under investigation.

\section{DISCUSSION}

Schizophrenia is a neurodevelopmental disorder associated with persistent symptomatology, severe functional disability, and residual morbidity characteristic of neurodegenerative brain diseases. The illness begins with genetic susceptibility and generally expresses itself after puberty through subtle changes that begin the prodromal stage. Symptoms get progressively worse and tend to become more resistant to treatment with each relapse. Evidence for a neuroprotective effect of some forms of early treatment is beginning to emerge. While the underlying mechanisms remain $[40,41]$. Studies that have examined markers of apoptosis and levels of apoptotic regulatory proteins in postmortem schizophrenia brain tissue indicated a dysfunction of apoptosis in several cortical regions in schizophrenia, including evidence that vulnerability of apoptosis is increased [42,43]. Although the exact role of apoptosis in schizophrenia remains uncertain, the potential involvement of non-lethal localized apoptosis is intriguing, especially in the earlier stages of the illness [44]. The continued clinical improvement of schizophrenic patients was surprising in light of their previous deterioration; the clinical improvement appears to be at least partly related to treatment with minocycline. Ahuja et al. described that it is possible that minocycline acted as a functional NMDA antagonist and helped improved the catatonic symptoms when used as an adjunct to antipsychotic medication [45]. Furthermore, minocycline appears to be safe for use in patients with advanced schizophrenia, although the precise mechanism of action of this agent remains unclear. The present results raise the possibility that minocycline may have effects beyond its action as an antiapoptotic agent [46-49].

\section{CONCLUSION}

Minocycline has demonstrated efficacy in treatment of schizophrenic patients in both open-label study, in two case reports, and randomized double-blind study. Moreover, preclinical finding are consistent with an antipsychotic-like profile of this molecule. Minocycline is also well tolerated. Minocycline counteracts apoptosis and inflammantion that are both common features in neurodegeneration, that minocycline has other therapeutic potential other than schizophrenia and/or that neurodegeneration has relevance to the pathophysiology of schizophrenia. The mechanism of action of minocycline that might be related to its antipsychotic potential appears to involve inhibition of apoptosis and inflammation. Furthermore, minocycline affords the possibility to be combined with other pharmacological molecules and shows promising properties for transplantation and gene therapy. However, it remains important to conduct further experimental studies on various relevant models to determine its activity upon caspase-independent degeneration, its long-term-effect on brain metabolism and also to precisely evaluate the additional potential benefit it could afford for cellular and molecules.

\section{REFERENCES}

[1] Yong, V.W., Wells, J., Giuliani, F., Casha, S., Power, C. and Metz, L.M. (2004) The promise of minocycline in neurology. The Lancet Neurology, 3, 744-751. doi:10.1016/S1474-4422(04)00937-8

[2] Conley, R.R. and Buchanan, R.V. (1997) Evaluation of treatment-resistant schizophrenia. Schizophrenia Bulletin, 23, 663-674. doi:10.1093/schbul/23.4.663 
[3] Miyaoka, T., Yasukawa, R., Yasuda, H., Hayashida, M., Inagaki, T. and Horiguchi, J. (2007) Possible antipsychotic effects of minocycline in patients with schizophrenia. Progress in Neuro-Psychopharmacology and Biological Psychiatry, 31, 304-307.

doi:10.1016/j.pnpbp.2006.08.013

[4] Miyaoka, T., Yasukawa, R., Yasuda, H., Hayashida, M., Inagaki, T. and Horiguchi, J. (2008) Minocycline as adjunctive therapy for schizophrenia: An open-label study. Clinical Neuropharmacology, 31, 287-292.

[5] Levkovitz, Y., Mendlovich, S., Riwkes, S., Braw, Y., Levkovitch-Verbin, H., Gal, G., Fenning, S., Treves, I. and Kron, S. (2010) A double-blind, randomized study of minocycline for the treatment of negative and cognitive symptoms in ealy-phase schizophrenia. Journal of Clinical Psychiatry, 71, 138-149.

doi:10.4088/JCP.08m04666yel

[6] Chaudhry, I.B., Hallak, J., Husain, N., Minhas, F.A., Stirling, J., Richardson, P., Dursun, S., Dunn, G. and Deakin, B. (2012) Minocycline benefits negative symptoms in early schizophrenia: a randomized double-blind placebo-controlled clinical trial in patients on standard treatment. Journal of Psychopharmacology (in press). doi:10.1177/0269881112444941

[7] Good, M.L. and Hussey, D.L. (2003) Minocycline: Stain devil? British Journal of Dermatology, 149, 237-239. doi:10.1046/j.1365-2133.2003.05497.x

[8] Colovic, M. and Caccia, S. (2003) Liquid chromatographic determination of minocycline in brain-toplasma distribution studies in the rat. Journal of Chromatography $B, 791,337-343$. doi:10.1016/S1570-0232(03)00247-2

[9] Bonelli, R.M., Heuberger, C. and Reisecker, F. (2003) Minocycline for Huntington's disease: An open label study. Neurology, 60, 883-884. doi:10.1212/01.WNL.0000049936.85487.7A

[10] Shapiro, L.E., Knowles, S.R. and Shear, N.H. (1997) Comparative safety of tetracycline, minocycline, and doxycycline. Archives of Dermatology, 133, 1224-1230. doi:10.1001/archderm.1997.03890460044005

[11] Gottlieb, A. (1997) Safety of minocycline for acne. Lancet, 349, 374. doi:10.1016/S0140-6736(97)80006-2

[12] Dommeguess, M.A., Plaisant, F., Vermey, C. and Gressens, O. (2003) Early microglial activation following neonatal excitoxic brain damage in mice: A potential target for neuroprotection. Neuroscience, 121, 619-628. doi:10.1016/S0306-4522(03)00558-X

[13] Yrjanheikki, J., Keinanen, R., Pellika, M., Hokfelt, T. and Hoistinaho, J. (1998) Tetracyclines inhibit microglial activation and are neuroprotective in global brain ischemia. Proceedings of the National Academy of Sciences, 95, 15769-15774. doi:10.1073/pnas.95.26.15769

[14] Yrjanheikki, J.,Tikka, T., Keinanen, R., Goldstein, G., Chan, P.H. and Koistinaho, J. (1999) A tetracycline derivative, minocycline, reduces inflammation and protects against focal cerebral ischemia with a wide therapeutic window. Proceedings of the National Academy of Sciences, 96, 13496-13500. doi:10.1073/pnas.96.23.13496

[15] Tikka, T., Fiebich, B.L., Goldsteins, G., Keinnanen, R. and Koistinaho, J. (2001) Minocycline, a tetracycline derivative, is neuroprotective against excitotoxicity by inhibiting activation and proliferation of microglia. The Journal of Neuroscience, 21, 2580-2588.

[16] Matsuki, S., Iuchi, Y., Ikeda, Y., Sasagawa, I., Tomita, Y. and Fujii, J. (2003) Suppression of cyctochrome c release and apoptosis in testes with heart stress by minocycline. Biochemical and Biophysical Research Communications, 312, 843-849. doi:10.1016/j.bbrc.2003.10.191

[17] Tikka, T.M. and Koistinaho, J.E. (2001) Minocycline provides neuroprotection against N-methyl-D-aspartate neurotoxicity by inhibiting microglia. The Journal of Immunology, 166, 7527-7533.

[18] Zhang, S.C., Goetz, B.D. and Duncan, I.D. (2003) Supression of activated microglia promotes survival and function of transplanted oligodendroglial progenitors. Glia, 41, 191-198. doi:10.1002/glia.10172

[19] Ekdahl, C.T., Claasen, J.H., Bonde, S., Kokaia, Z. and Lindvall, O. (2003) Inflammation is detrimental for neurogenesis in adult brain. Proceedings of the National Academy of Sciences, 100, 13632-13637. doi:10.1073/pnas.2234031100

[20] Stiring, D.P., Khodarahmi, K., Liu, J., et al. (2004) Minocycline treatment reduces delayed oligodendrocyte death, attenuates axonal dieback, and improves functional outcome after spinal cord injury. The Journal of Neuroscience, 24, 2182-2190. doi:10.1523/JNEUROSCI.5275-03.2004

[21] Arvin, K.L., Han, B.H., Du, Y., Lin, S.Z., Paul, S.M. and Holzman, D.M. (2002) Minocycline markedly protects the neonatal brain against hypoxic-ischemic injury. Annals of Neurology, 52, 54-61. doi:10.1002/ana.10242

[22] Zhu, S., Stavrovskaya, I.G., Drozda, M., et al. (2002) Minocycline inhibits cytochrome c release and delayes progression of amyotrophic lateral sclerosis in mice. $\mathrm{Na}$ ture, 417, 74-78. doi:10.1038/417074a

[23] Wang, X., Zhu, S., Drozda, M., et al. (2000) Minocycline inhibits caspase- 1 and caspase- 3 expression and delayes mortality in a transgenic mouse model of Huntington disease, Nature Medicine, 6, 797-801. doi:10.1038/77528

[24] Wang, J., Wei, Q., Wang, C.Y., Hill, W.D., Hess, D.C. and Dong, Z. (2004) Minocycline up-regulates Bcl-2 and protects against cell death in mitochondria. The Journal of Biological Chemistry, 279, 19948-19954. doi:10.1074/jbc.M313629200

[25] Gabler, W.L., Amith, J. and Tsukuda, N. (1992) Comparison of deoxycycline and a chemically modified tetracycline inhibition of leukocyte functions. Research Communications in Chemical Pathology \& Pharmacology, 78, 151-160.

[26] Amin, A.R., Attur, M.G., Thakker, G.D., et al. (1996) A novel mechanism of action of tetracyclines: Effect on nitric oxide synthases. Proceedings of the National Academy of Sciences, 93, 14014-14019. doi:10.1073/pnas.93.24.14014

[27] Young, V.W., Power, C., Forsyth, P. and Edwards, D.R. (2001) Metalloproteinases in biology and pathology of the nervous system. Nature Reviews Neuroscience, 2, 502-511. doi:10.1038/35081571 
[28] Golub, L.M., Ramamurthy, N., McNamara, T.F., et al. (1984) Tetracyclines inhibit tissue collagenase activity. A new mechanism in the treatment of periodontal disease. Journal of Periodontal Research, 19, 651-655. doi:10.1111/j.1600-0765.1984.tb01334.x

[29] Paemen, L., Martens, E., Norga, K., et al. (1996) The gelatinase inhibitory activity of tetracyclines and chemically modified tetracycline analogues as measured by a novel microtiter assay for inhibitiors. Biochemical Pharmacology, 52, 105-111. doi:10.1016/0006-2952(96)00168-2

[30] Power, C., Henry, S., Del Bigio, N.R., et al. (2003) Untracerebral hemorrhagew induces macrophage activation and matrix metalloproteinases. Annals of Neurology, 53, 731-742. doi:10.1002/ana.10553

[31] Kloppenburg, M., Verweij, C.L., Miltenburg, A.M., et al. (1995) The influence of tetracyclines on T cell activation. Clinical and Experimental Immunology, 102, 635-641. doi:10.1111/j.1365-2249.1995.tb03864.x

[32] Kloppenburg, M., Brinkman, B.M., de Rooji-Dijk, N.H., et al., (1996) The tetracycline derivate minocycline differentially affects cytokine production by monocytes and T lymphocytes. Antimicrobial Agents and Chemotherapy, 40, 934- 940.

[33] Hayashida, M., Miyaoka, T., Tsuchie, K., Yasuda, H., Wake, R., Nishida, A., Inagaki, T., Toga, T., Nagami, H., Oda, T. and Horiguchi, J. (2009) Hyperbilirubinemia-related behavioral and neuropathological changes in rats: A possible schizophrenia animal model. Progress in Neuro-Psychopharmacology \& Biological Psychiatry, 33, 581-588. doi:10.1016/j.pnpbp.2009.02.013

[34] Liaury, K., Miyaoka, T., Tsumori, T., Furuya, M., Wake, R., Ieda, M., Tsuchie, K., Taki, M., Ishihara, K., Tanra, A.J. and Horiguchi, J. (2012) Morphological features of microglial cells in the hippocampal dentate gyrus of Gunn rat: A possible schizophrenia animal model. Journal of Neuroinflammation, 9, 56. doi:10.1186/1742-2094-9-56

[35] Levkovitz, Y., Levi, U., Braw, Y. and Cohen, H. (2007) Minocycline, a second-generation tetracycline, as a neuroprotective agent in an animal model of schizophrenia. Brain Research, 1154, 154-162.

[36] Zhang, L., Shirayama, Y., Iyo, M. and Hashimoto, K. (2007) Minocycline attenuates hyperlocomotion and prepulse inhibition deficits in mice after administration of the NMDA receptor antagonist dizocilpine. Neuropsychopharmacology, 32, 2004-2010. doi:10.1038/sj.npp.1301313

[37] Mizoguchi, H., Takuma, K., Fukakusa, A., Ito, Y., Nakatani, A., Ibi, D., Kim, H.C. and Yamada, K. (2007) Improvement by minocycline of methamphetamine-induced impairment of recognition memory in mice. Psy- chopharmacology, 196, 233-241. doi:10.1007/s00213-007-0955-0

[38] Kay, S.R., Fiszbein, A. and Opler, L.A. (1987) The positive and negative syndrome scale (PANSS) for schizophrenia. Schizophrenia Bulletin, 13, 261-276.

[39] American Psychiatric Association (1994) Diagnostic and Statistical Manual of Mental Disorders, 1994; 4th Edition, American Psychiatric Press, Washington DC.

[40] Weinberger, D.R. (1987) Implications of normal brain development for the pathogenesis of schizophrenia. Archives of General Psychiatry, 44, 660-669. doi:10.1001/archpsyc.1987.01800190080012

[41] Waddington, J.L. (1993) Schizophrenia: Developmental neuro- science and pathology. Lancet, 341, 531-536. doi:10.1016/0140-6736(93)90288-R

[42] Arnold, S.E., Trojanowski, J.Q., Gur, R.E., Blackwell, P., Han, L.Y. and Choi, C. (1998) Absence of neurodegeneration and neural injury in the cerebral cortex in a sample of elderly patients with schizophrenia. Archives of General Psychiatry, 55, 225-232.

[43] Harrison, P.J. and Weinberger, D.R. (2005) Schizophrenia genes, gene expression, and neuropathology: On the matter of their convergence. Molecular Psychiatry, 10, 40-68. doi:10.1038/sj.mp.4001558

[44] German, T.T., Tong, R.I., Gilmore, J.H., Lieberman, J.A. and Jarskog, L.F. (2004) Regulation of apoptosis by typical and atypical antipsychotics in rat frontal cortex. European Neuropsychopharmcology, 55, 214.

[45] Ahuja, N. and Carroll, B.T. (2007) Possible anti-catatonic effects of minocycline in patients with schizophrenia. Progress in Neuro-Psychopharmacology and Biological Psychiatry, 31, 968-969. doi:10.1016/j.pnpbp.2007.01.018

[46] Kempermann, G., Krebs, J. and Fabel, K. (2008) The contribution of failing adult hippocampal neurogenesis to psychiatric disorders. Current Opinion in Psychiatry, 21, 290-295. doi:10.1097/YCO.0b013e3282fad375

[47] Lei, G., Xia, Y. and Johnson, K.M. (2008) The role of Akt-GSK-3beta signaling and synaptic strength in phencyclidine-induced neurodegeneration. Neuropsychopharmacology, 33, 1343-1353. doi:10.1038/sj.npp.1301511

[48] Csernansky, J.G. (2007) Neurodegeneration in schizophrenia: evidence from in vivo neuroimaging studies. Scientific World Journal, 7, 135-143. doi:10.1100/tsw.2007.47

[49] Lieberman, et al. (2007) Neuroprotection: a therapeutic strategy to prevent deterioration associated with schizophrenia. CNS Spectrums, 3, 1-13; 